\title{
Dense astrophysical plasmas
}

\author{
G Chabrier $\dagger$, F Douchin $\dagger$ and A Y Potekhin $\ddagger$ \\ $\dagger$ Ecole Normale Supérieure de Lyon, C.R.A.L.(UMR 5574 CNRS) \\ 69364 Lyon Cedex 07, France \\ $\ddagger$ Ioffe Physico-Technical Institute, 194021 St Petersburg, Russia
}

\begin{abstract}
.
We briefly examine the properties of dense plasmas characteristic of the atmospheres of neutron stars and of the interior of massive white dwarfs. These astrophysical bodies are natural laboratories to study respectively the problem of pressure ionization of hydrogen in a strong magnetic field and the crystallization of the quantum one-component-plasma at finite temperature.
\end{abstract}


prepared for "Liquid state theory : from white dwarfs to colloids"

An international conference on the occasion

of Prof. Jean-Pierre Hansen's 60th birthday

Les Houches April 1-5, 2002

\section{Introduction}

The quest for an accurate description of the thermodynamics properties of dense plasmas has represented a thriving domain of research since the seminal review by Baus and Hansen (1980). The interiors of many astrophysical bodies are characterized by strongly correlated ionic and electronic plasmas, with respective classical and quantum coupling parameters $\Gamma_{\mathrm{i}}=\beta\left(Z_{\mathrm{i}} e\right)^{2} / a_{\mathrm{i}}$ and $r_{s}=a_{\mathrm{i}} /\left(a_{0} Z_{\mathrm{i}}^{1 / 3}\right)$ varying over several decades. Here, $a_{0}=\hbar^{2} /\left(m_{e} e^{2}\right)$ denotes the electronic Bohr radius, $a_{\mathrm{i}}=\left(3 N_{\mathrm{i}} / 4 \pi V\right)^{1 / 3}$ is the mean inter-ionic distance, and $\beta \equiv 1 /\left(k_{\mathrm{B}} T\right)$. The correct description of the thermodynamic properties of these astrophysical bodies, which determine their mechanical and thermal properties, thus requires the knowledge of the equation of state (EOS) of such plasmas. In this short review, we focus on the case of neutron stars (NS) and massive white dwarfs (WD), which exhibit two particularly interesting problems in the statistical physics of dense matter.

\section{Ionization equilibrium of a hydrogen plasma in strong magnetic fields}

Most of neutron stars are characterized by magnetic fields $B \sim 10^{11}-10^{13} \mathrm{G}$, whereas some of them (so-called magnetars) are thought to have $B \sim 10^{14}-10^{15} \mathrm{G}$. Although huge by the terrestrial standards, the magnetic energy of a NS, $\sim R^{3} B^{2} / 6$, represents only a tiny fraction of its gravitational binding energy $E_{G} \sim G M^{2} / R^{2}$.

The photospheric properties of a NS are characterized by temperatures $T \simeq 10^{5}$ $10^{7} \mathrm{~K}$ (depending on the age and mass of the star) and densities $\rho \simeq 10^{-2}-10^{4} \mathrm{~g} \mathrm{~cm}^{-3}$ (depending on $T$ and $B$ ). The cooling rates of these stars are entirely determined by the relationship between the photospheric and the interior temperature profiles. The emitted spectra of these stars can be strongly affected by the presence of bound species in the photosphere. Since the atmosphere can be composed essentially of hydrogen accreted from either the interstellar medium, the supernova remnant or a close companion, the determination of the temperature profiles and spectra thus requires an accurate description of hydrogen ionization in a strong magnetic field.

The quantum-mechanical properties of protons, free electrons and bound species (hydrogen atoms and molecules) are strongly modified by the field, which thereby affects the thermodynamic properties of the plasma. The properties of matter in a magnetic field under NS conditions have been reviewed recently by Ventura and Potekhin (2001) and Lai (2001). We refer the reader to these reviews for the detailed descriptions of these properties, and only the most recent results will be outlined in the present paper.

The transverse motion of electrons in a magnetic field is quantized into Landau 
levels. The energy of the $n$th Landau level of the electron (without the rest energy) is $m_{e} c^{2}(\sqrt{1+2 b n}-1)$, which becomes $\hbar \omega_{c} n$ in the non-relativistic limit, where

$$
\hbar \omega_{c}=\hbar \frac{e B}{m_{e} c}=11.577 B_{12} \mathrm{keV}
$$

is the electron cyclotron energy,

$$
b=\hbar \omega_{c} / m_{e} c^{2}=B_{12} / 44.14
$$

is the field strength in the relativistic units, and $B_{12}=B /\left(10^{12} \mathrm{G}\right)$ is a typical magneticfield scale for NS conditions.

The atomic unit for the magnetic-field strength is set by $\hbar \omega_{c}=e^{2} / a_{0}$, i.e. $B_{0}=\left(m_{e} c / \hbar e\right) \times\left(e^{2} / a_{0}\right)=2.35 \times 10^{9} \mathrm{G}$. It is convenient to define a dimensionless magnetic-field strength

$$
\gamma=B / B_{0}=b / \alpha_{f}^{2}
$$

where $\alpha_{f}$ is the fine structure constant.

For $\gamma \gg 1$, as encountered in NS conditions, the electron cyclotron energy is much larger than the typical Coulomb energy, so that the properties of interacting particles, protons, atoms, molecules, are strongly affected by the field. The ground-state atomic and molecular binding energies increase with $B$ as $\sim \ln ^{2} \gamma$. The atom in a strong magnetic field is compressed in the transverse directions to the radius $\sim a_{\mathrm{m}}$, where

$$
a_{\mathrm{m}}=(\hbar c / e B)^{1 / 2}=\gamma^{-1 / 2} a_{0}=2.56 \times 10^{-10} B_{12}^{-1 / 2} \mathrm{~cm}
$$

is the quantum magnetic length, which becomes the natural length unit in the plasma instead of $a_{0}$.

The thermal motion of atoms causes the Stark effect due to the electric field induced in the comoving frame of reference. At $\gamma \gg 1$, this effect strongly modifies the atomic properties: the atom becomes asymmetric, and its binding energy and oscillator strengths depend on the velocity (Potekhin 1994). Two classes of the atomic states arise: so-called centered and decentered states; for the latter ones the electron-proton separation is large and the binding energy relatively small (Vincke et al 1992; Potekhin 1994).

The formation of molecules is also strongly modified in a strong magnetic field. Because of the alignment of the electron spins antiparallel to the field, two atoms in their ground state $(m=0)$ do not bind together, because of the Pauli exclusion principle. One of the two $\mathrm{H}$ atoms has to be excited in the $m=-1$ state to form the ground state of the $\mathrm{H}_{2}$ molecule, which then forms by covalent bonding (e.g., Lai, 2001).

As long as $T \ll \hbar \omega_{c} / k_{\mathrm{B}}=1.343 \times 10^{8} B_{12} \mathrm{~K}$ and $\rho \ll \rho_{B} \approx 7.1 \times 10^{3} B_{12}^{3 / 2} \mathrm{~g} \mathrm{~cm}^{-3}$, the electron cyclotron energy $\hbar \omega_{c}$ exceeds both the thermal energy $k_{\mathrm{B}} T$ and the electron Fermi energy $k_{\mathrm{B}} T_{\mathrm{F}}$, so that the electrons are mostly in the Landau ground state, - i.e., the field is strongly quantizing. In this case, typical for the NS photospheres, the electron spins are aligned antiparallel to the field.

Proton motion is also quantized by the magnetic field, but the corresponding cyclotron energy is smaller, $\hbar \omega_{c p}=\hbar \omega_{c} m_{e} / m_{p}$. 

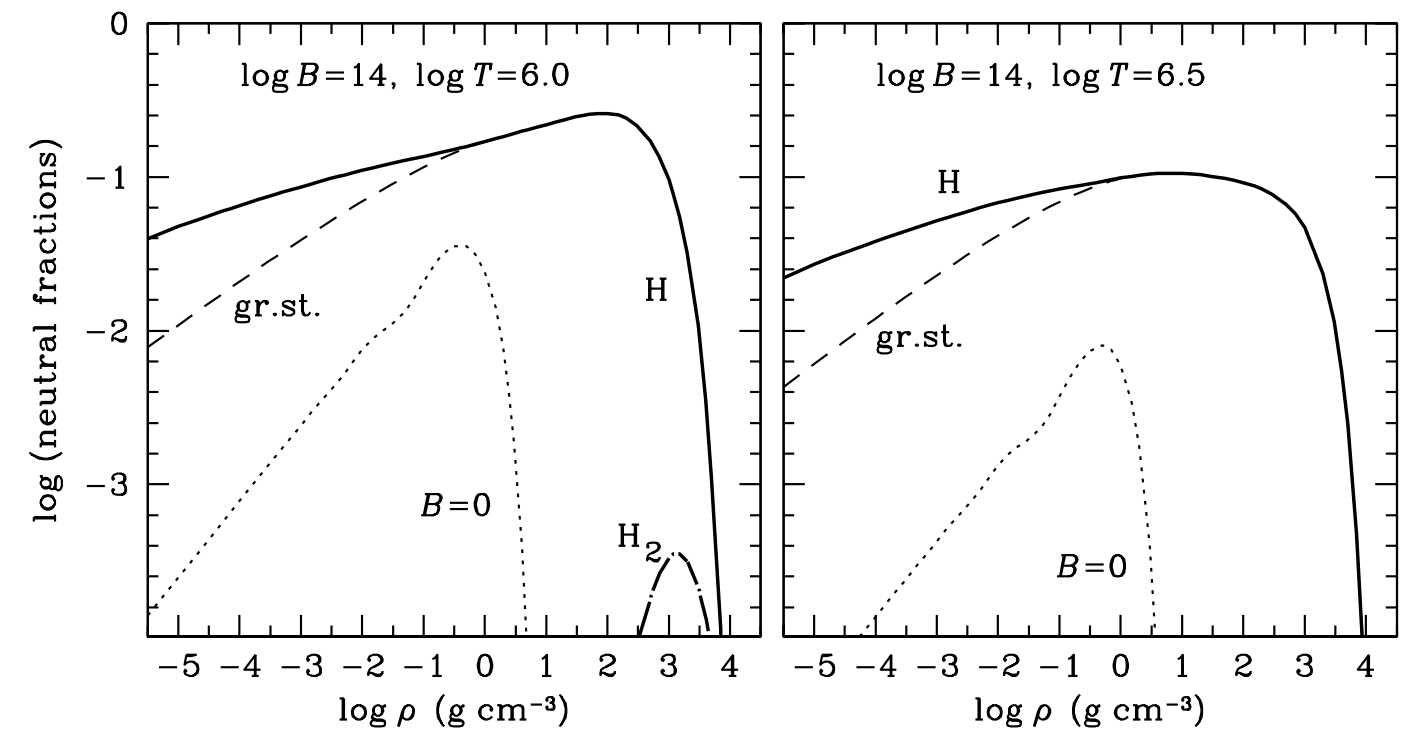

Figure 1. Ionization isotherms at $B=10^{14} \mathrm{G}$ and two values of $T$. Solid lines: total fraction of atoms $f_{\mathrm{H}}=n_{\mathrm{H}} / n_{0}$; dash-lines: fractions of ground-state atoms; dot-dashed lines: molecular fraction $f_{\mathrm{H}_{2}}=2 n_{\mathrm{H}_{2}} / n_{0}$, where $n_{0}$ is the total number density of protons (free and bound). Dotted lines: zero-field case

Quantum-mechanical calculations of the binding energies and wave functions of hydrogen atoms in any states of motion in the strong magnetic fields have been carried out only recently (Vincke et al 1992; Potekhin, 1994). Using these results, Potekhin, Chabrier and Shibanov (1999) derived a model which describes the thermodynamics of an interacting $\left(\mathrm{H}_{2}, \mathrm{H}, \mathrm{H}^{+}, \mathrm{e}^{-}\right)$plasma in a strong magnetic field. This model is based on the framework of the free energy model developed by Saumon and Chabrier (1991, 1992 ) for pressure ionization of hydrogen at $B=0$, but generalizes it to the strong- $B$ case, taking into account the quantum-mechanical effects caused by the thermal motion of atoms across the magnetic field.

Potekhin et al (1999) calculated the ionization equilibrium and EOS at $7 \times 10^{11} \mathrm{G} \leq$ $B \leq 3 \times 10^{13} \mathrm{G}$. In this paper, we extend these calculations up to $B=10^{14} \mathrm{G}$, typical for the magnetars. We calculate the number densities of atoms $\left(n_{\mathrm{H}}\right)$ and molecules $\left(n_{\mathrm{H}_{2}}\right)$ from the equations

$n_{\mathrm{H}}=n_{e}^{2} \frac{\lambda_{p} \lambda_{e}\left(2 \pi a_{\mathrm{m}}^{2}\right)^{2}}{\lambda_{\mathrm{H}}^{3}}\left[1-e^{-\beta \hbar \omega_{c p}}\right] Z_{w} \mathrm{e}^{\Lambda}, \quad n_{\mathrm{H}_{2}}=n_{\mathrm{H}}^{2}\left(\lambda_{\mathrm{H}} \sqrt{2}\right)^{3} Z_{w 2} / Z_{w}^{2}$,

where $n_{e}$ is the electron number density, $\lambda_{j}=\left(2 \pi \beta \hbar^{2} / m_{j}\right)^{1 / 2}$ is the thermal wavelength of the particle $j(j=e, p, \mathrm{H}), Z_{w}$ and $Z_{w 2}$ are the internal partition functions for $\mathrm{H}$ and $\mathrm{H}_{2}$, respectively, and $\Lambda$ is a correction factor, which takes into account electron degeneracy and filling of the excited Landau levels. The formulae for $Z_{w}, Z_{w 2}$ and $\Lambda$ are given in Potekhin et al (1999). 

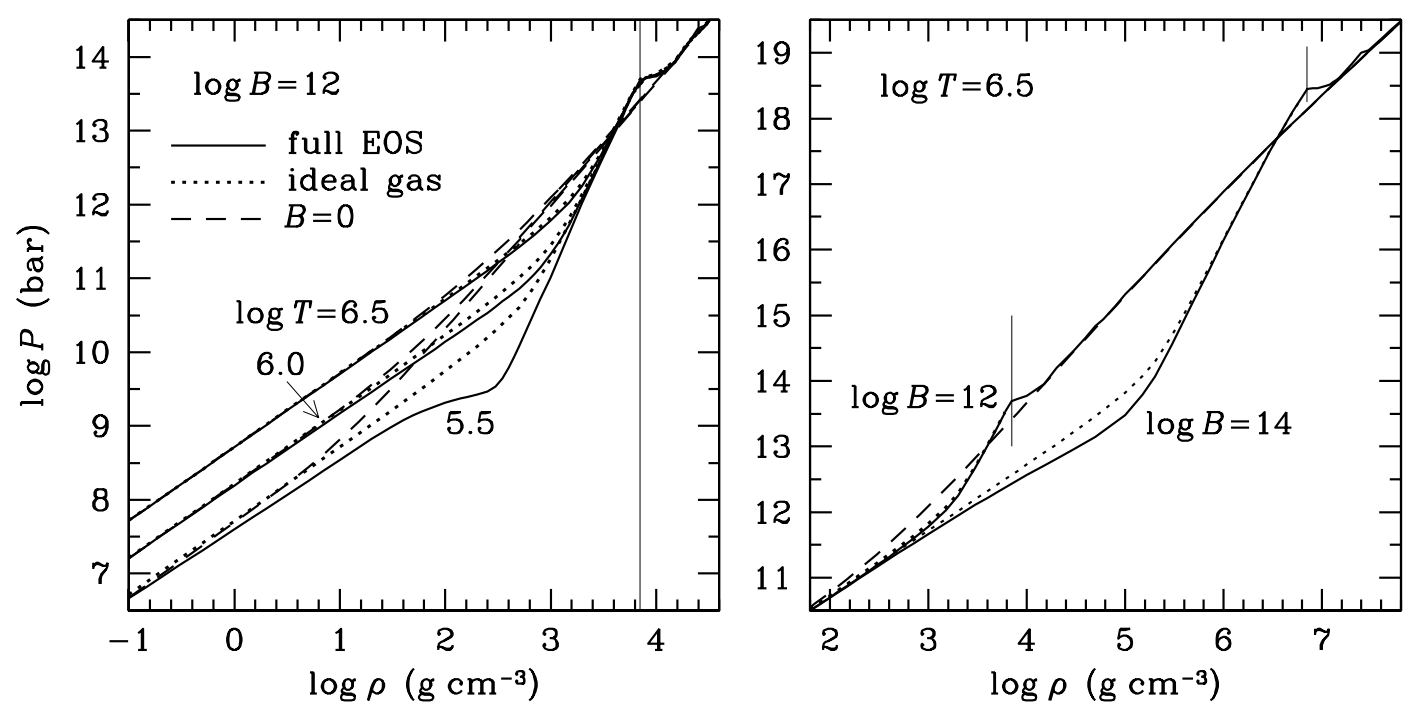

Figure 2. EOS of partially ionized atomic hydrogen in the strong magnetic field (solid lines) compared with the EOS of a fully ionized ideal electron-proton plasma (dotted lines) and the EOS of a partially ionized hydrogen at $B=0$ (dashed lines). Left panel: $B=10^{12} \mathrm{G}, \log T[\mathrm{~K}]=6.5,6.0$, and 5.5. Right panel: $\log T[\mathrm{~K}]=6.5, B=10^{12} \mathrm{G}$ and $10^{14} \mathrm{G}$. The vertical lines correspond to the density above which excited Landau levels become populated

Figure 1 displays the logarithm of the fraction of $\mathrm{H}$ and $\mathrm{H}_{2}$ for a magnetic field $B=10^{14} \mathrm{G}$, for two isotherms. The solid line represents the total fraction of atoms $f_{\mathrm{H}}=n_{\mathrm{H}} / n_{0}\left(n_{0}=n_{\mathrm{H}}+n_{p}+2 n_{\mathrm{H}_{2}}\right)$ in all quantum states, whereas the dashed line shows the fraction of atoms in the ground state. The dotted line displays the zero-field result. As seen in this figure, the strong magnetic field favours atomic and molecular recombination. Since the binding energies of atoms and molecules increase and $T_{\mathrm{F}}$ decreases in the strongly quantizing magnetic field, pressure ionization occurs at much larger densities than for the field-free case.

Figure 2 displays the resulting EOS. The left panel displays three isotherms at $B=10^{12} \mathrm{G}$, typical of ordinary NS. On the right panel, we compare the isotherm $T=10^{6.5} \mathrm{~K}$ for $B=10^{12} \mathrm{G}$ and for the superstrong field $B=10^{14} \mathrm{G}$. As expected from the previous discussion, the EOS in a strong magnetic field differs significantly from the field-free case in the region of partial ionization. At very high-density, excited Landau levels become populated, due to the increase of the Fermi energy, and the zero-field case is recovered. 


\section{Crystallization of white dwarf cores}

Massive white dwarfs $\left(1.2 \mathrm{M}_{\odot} \leq M \leq 1.4 \mathrm{M}_{\odot}=M_{\mathrm{Ch}}\right.$, where $M_{\mathrm{Ch}}$ denotes the Chandraskhar mass) are $\mathrm{C}^{6+} / \mathrm{O}^{8+}$ plasmas with central density and temperatures $\rho_{\mathrm{c}} \approx 10^{8}-10^{9} \mathrm{~g} \mathrm{~cm}^{-3}, T_{\mathrm{c}} \approx 10^{6} \mathrm{~K}$. Under these conditions, the ion zero-point energy $E_{0} \propto$ $\hbar \Omega_{P}$, where $\Omega_{P}=\left(3 Z_{\mathrm{i}}^{2} e^{2} / M_{\mathrm{i}} a_{\mathrm{i}}^{3}\right)^{1 / 2}$ is the ion plasma frequency, exceeds the classical thermal energy $k_{\mathrm{B}} T$ (Chabrier et al 1992), i.e. $\eta=\hbar \Omega_{P} / k_{\mathrm{B}} T \gg 1$. Collective diffraction effects thus modify the classical Coulomb interaction. The melting values of the coupling parameters $\Gamma_{\mathrm{m}}$ for the classical OCP and $R_{S, \mathrm{~m}}$, where $R_{S}=a_{\mathrm{i}} /\left(\hbar^{2} / M_{\mathrm{i}} Z_{\mathrm{i}}^{2} e^{2}\right)$ is the ion quantum plasma parameter, for the quantum jellium model at zero-temperature, have been firmly established: $\Gamma_{\mathrm{m}}=175$ (Potekhin and Chabrier 2000), and $R_{S, \mathrm{~m}}=160$ for bosons, $R_{S, \mathrm{~m}}=100$ for fermions (Ceperley and Alder 1980). The melting curve at finite temperature, however, i.e., at $\eta \neq 0$ and $T \neq 0$, still remains poorly determined.

A simplified determination of this curve is based on the Lindeman critical parameter interpolation between the zero-temperature and the classical melting values (Mochkovitch and Hansen 1979; Chabrier 1993). These calculations, however, are based on a harmonic description of the phonon mode spectrum and thus do not include nonharmonic effects. More recently, Jones and Ceperley (1996) performed Path Integral Monte Carlo (PIMC) simulations to try to determine the phase diagram more correctly. They found a maximum melting temperature almost a factor of 2 larger than the one determined by Chabrier (1993).

In this paper, we present preliminary results based on similar PIMC calculations at finite temperature. The system under consideration consists of $N$ identical, but distinguishable particles (Boltzmannions) with a mass $M$ and charge $Z e$ in a volume $V$ at temperature $T$.

The partition function of the quantum system is given by the trace of the $N$ body density matrix $\rho_{N}\left(\vec{R}, \vec{R}^{\prime} ; \beta\right)=\left\langle\vec{R}\left|e^{-\beta H_{N}}\right| \vec{R}^{\prime}\right\rangle$. Here $\vec{R}$ denotes the $3 N$ coordinates $\left\{\vec{r}_{i}\right\}_{i=1, \ldots, N}$. Using the Trotter formula, this partition function can be rewritten exactly in terms of the density matrix $\rho_{N}\left(\vec{R}, \vec{R}^{\prime} ; \tau\right)$ with $\tau=\beta / P$ (Ceperley 1995) as

$$
Q_{N, V, T}(\beta)=\int \Pi_{\alpha=1}^{P} d \vec{R}_{\alpha} \rho_{N}\left(\vec{R}_{\alpha}, \vec{R}_{\alpha+1} ; \tau\right)
$$

In the $P \rightarrow \infty$ limit, the exact density matrix

$$
e^{-\beta H_{N}}=\lim _{P \rightarrow \infty}\left[e^{-\frac{\beta}{P} K_{N}} \times e^{-\frac{\beta}{P} V_{N}}\right]^{P}
$$

is recovered by using the approximate expression

$$
\rho_{N}\left(\vec{R}_{\alpha}, \vec{R}_{\beta} ; \tau\right)=\rho_{N}^{0}\left(\vec{R}_{\alpha}, \vec{R}_{\beta} ; \tau\right) \exp \left[-\frac{\tau}{2}\left\{V^{c}\left(\vec{R}_{\alpha}\right)+V^{c}\left(\vec{R}_{\beta}\right)\right\}\right],
$$

where $K_{N}$ and $V_{N}$ denote the kinetic and potential parts of the Hamiltonian, respectively, $\rho_{N}^{0}\left(\vec{R}_{\alpha}, \vec{R}_{\beta} ; \tau\right)=(4 \pi \tau)^{-3 N / 2} \exp \left[-\left(\vec{R}_{\alpha}-\vec{R}_{\beta}\right)^{2}\right] /(4 \tau)$ is the density matrix of free particles, and $V^{c}$ is the classical potential energy of the system.

In terms of the path integral formalism, the particle is defined by its trajectory in "imaginary time" $P \tau$, through "polymers" composed of $P$ monomers connected by "strings" of stiffness $M_{\mathrm{i}} / \hbar \tau$. 


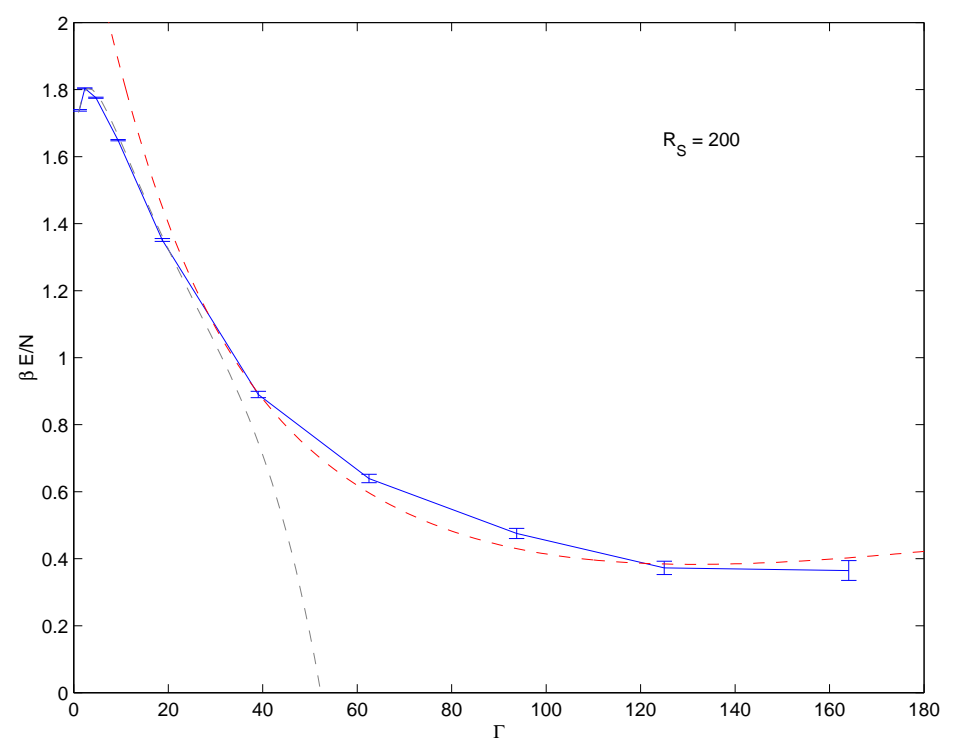

Figure 3. Internal energy of a quantum OCP at $R_{S}=200$. Solid lines: PIMC calculations; left dash-line: Hansen-Vieillefosse (1975) $\hbar^{4}$-expansion; right dash line: analytical fit of the energy at large $\Gamma$ (Douchin and Chabrier 2002).

We have performed simulations along three isochores $R_{S}=1200,350,200$ and five isotherms on each isochore around the estimated melting temperature. In order to estimate the finite-size effects, we used 16, 54, 128 and 250 particles. For each simulation we take $\eta / P=0.05-0.1$. We then parametrized the fluid and solid internal energies, including the finite-size corrections. The melting temperature was estimated both from free energy comparison and from a dynamical criterion, namely the mean square displacement in unit of the nearest-neighbour distance $\left(\left\langle\delta r^{2}\right\rangle / d^{2}\right)^{1 / 2}$. This value is finite in a solid and diverges in a fluid.

Figure 3 displays the internal energy of the quantum fluid for $R_{S}=200$. The solid line is the result of the PIMC simulation, with $N=54$ particles. The dash line displays the Hansen-Vieillefosse (1975) $\hbar^{4}$-expansion, which diverges when the thermal wavelength $\lambda_{\mathrm{i}}$ is of the order of the inter-ionic distance $a_{\mathrm{i}}$. At large $\Gamma$, i.e. large $\eta$ for a given $R_{S}$, the energy tends towards the zero-temperature limit (Chabrier, 1993). The dash-line displays an analytical fit of the PIMC energy at large $\Gamma$ (Douchin and Chabrier, 2002).

Our preliminary results yield melting temperatures lower than the ones obtained by Jones and Ceperley (1996), about a factor of 2 for the maximum melting temperature. This difference stems from a better correction of finite-size effects, which are known to stabilize the solid. First of all, we explored more in detail the N-dependence of our PIMC simulations by conducting calculations with a larger number of particles (up to $\mathrm{N}=256$ ). Second of all, we found out that the dependence on $\Gamma$ and $R_{S}$ of the finitesize effects for the liquid phase is more complex than the simple one used by Jones 
and Ceperley (1996). This is particularly important for the extrapolated values of the energy around the turning point. The obtained maximum melting temperature and corresponding density are (Douchin and Chabrier, 2002)

$$
T_{\max }=8810 A Z^{4} \mathrm{~K} ; \quad \rho_{\mathrm{m}}=1280 A^{4} Z^{6} \mathrm{~g} \mathrm{~cm}^{-3} \text {, i.e., } R_{S} \approx 235 .
$$

These calculations show that, although anharmonic effects are non-negligible both in the solid and in the liquid phase, they are comparable in both phases and almost cancel out, so that the melting curve lies close to the one estimated from the harmonic spectrum.

\section{Conclusion}

In this short review, we have considered two different problems related to dense plasma physics as encountered under specific astrophysical conditions. Because of the presence of a strong magnetic field, the quantum internal levels of atoms and molecules are quantized in Landau orbits, and the field raises the binding energy of these species, favoring recombination over dissociation and ionization compared with the field-free case. This modifies the ionization equilibrium and EOS of the dense plasma.

We also considered the crystallization of a quantum fluid of Boltzmannions at finite temperature with PIMC simulations, taking into account the correction due to finitesize effects. We found that the crystallization diagram lies close to the one based on an interpolation of the Lindeman criterium between the classical and the zero-temperature limits. These preliminary results need to be confirmed by further calculations. Such work is under progress.

These two examples, and many other not mentioned in the present review, stress

the need for detailed calculations of the properties of dense plasmas under extreme conditions for astrophysical applications.

\section{Acknowledgment}

The work of A.P. was supported in part by RFBR Grants 02-02-17668 and 00-07-90183.

\section{References}

Baus M and Hansen J P 1980 Phys. Rep. 591

Ceperley D M 1995 Rev. Mod. Phys. 67279

Ceperley D M and Alder B J 1980 Phys. Rev. Lett. 45566

Chabrier G 1993 Astrophys. J. 414695

Chabrier G, Ashcroft N W and DeWitt H E 1992 Nature 36048

Douchin F and Chabrier G 2002 (in preparation)

Hansen J P and Vieillefosse P 1975 Phys. Lett. 53A 187

Jones M D and Ceperley D M 1996 Phys. Rev. Lett. 764572

Lai D 2001 Rev. Mod. Phys. 73729

Mochkovitch R and Hansen J P 1979 Phys. Lett. A73, 35

Potekhin A Y 1994 J. Phys. B: At. Mol. Opt. Phys. 271073 
Potekhin A Y and Chabrier G 2000 Phys. Rev. E 628554

Potekhin A Y, Chabrier G and Shibanov Yu A 1999 Phys. Rev. E 602193

Saumon D and Chabrier G 1991 Phys. Rev. A 44, 5122

Saumon D and Chabrier G 1992 Phys. Rev. A 46, 2084

Ventura J and Potekhin A Y 2001 The Neutron Star - Black Hole Connection, NATO Science Ser. C 567, C Kouveliotou, J Ventura and E P J van den Heuvel (Dordrecht: Kluwer) p 393

Vincke M, Le Dourneuf M and Baye D 1992 J. Phys. B: At. Mol. Opt. Phys. 252787 